\title{
TENDÊNCIA TEMPORAL DA MORTALIDADE POR SUICÍDIO NO ESTADO DA BAHIA
}

\author{
Érica Assunção Carmo ${ }^{1}$, Bárbara Santos Ribeiro ${ }^{1}$, Adriana Alves $\mathrm{Nery}^{2}$,Cezar Augusto Casotti ${ }^{3}$
}

\begin{abstract}
RESUMO: Objetivo: Analisar a tendência temporal da mortalidade por suicídio no estado da Bahia, segundo sexo e faixa etária, no período de 1996 a 2013. Métodos: Estudo de série temporal realizado com dados do Sistema de Informação sobre Mortalidade, com análise de tendência por meio da regressão de Prais-Winsten. Resultados: Identificou-se 5.693 óbitos por suicídio na Bahia, destes $81,4 \%(n=4632)$ eram homens e $46 \%$ ( $n=2619)$ do grupo etário de 20 a 39 anos. Quanto ao meio utilizado $58,6 \%(n=3336)$ foram decorrentes de lesões autoprovocadas por enforcamento/estrangulamento, seguidas pela autointoxicação por pesticidas e por produtos químicos $(15,9 \% /$ $\mathrm{n}=906$ ). Verificou-se tendência crescente para o coeficiente de mortalidade por suicídio no Estado, para ambos os sexos, e para os grupos etários de 20 a 39 anos, 40 a 59 anos e de 60 anos ou mais. Conclusão: O suicídio na Bahia predomina entre homens jovens, com tendência crescente para o coeficiente total e nos diferentes grupos populacionais.
\end{abstract}

DESCRITORES: Suicídio; Mortalidade; Estudos de séries temporais; Sistemas de informação; Estudos ecológicos.

\section{TIME TREND IN SUICIDE MORTALITY IN THE STATE OF BAHIA}

ABSTRACT: Objective: To analyze the time trend in suicide mortality in the state of Bahia according to gender and age range, in the 1996-2013 period. Methods: A time series study based on data from Brazil'a Mortality Information System (SIM), with trend analysis that uses Prais-Winsten regression. Results: Of the 5,393 people who died from suicide in Bahia, $81.4 \%(\mathrm{n}=4632)$ were men and $46 \%$ $(n=2619)$ were aged 20-39 years. As to the means used, 58.6\% $(n=3336)$ of the deaths were due to intentional self-harm by hanging and strangulation, followed by self-poisoning by pesticides and chemicals $(15.9 \% / n=906)$. There was an increasing trend in the coefficient of mortality by suicide in the State for both genders and for the groups of 20 to 39 years, 40 to 59 years and 60 years or more. Conclusion: Suicide in Bahia predominates among young males, with an increasing trend of the total coefficient and in the different population groups.

DESCRIPTORS: Suicide; Mortality; Time series studies; Information systems; Ecological studies.

\section{TENDENCIA TEMPORAL DE LA MORTALIDAD POR SUICIDO EN EL ESTADO DE BAHÍA}

RESUMEN: Objetivo: Analizar la tendencia temporal de la mortalidad por suicidio en el Estado de Bahía, según sexo y faja etaria, entre 1996 y 2013. Métodos: Estudio de serie temporal, realizado con datos del Sistema de Información sobre Mortalidad, con análisis de tendencia por regresión de Prais-Winsten. Resultados: Se identificaron 5.693 decesos por suicidio en Bahía;de ellos, 81,4\% (n=4.632) correspondía a hombres y $46 \%(n=2.619)$ pertenecía al segmento etario de 20 a 39 años. Respecto al medio utilizado, 58,6\% ( $n=3.336)$ derivaron de lesiones autoinfligidas, por ahorcamiento/estrangulamiento, seguidas por autointoxicación con pesticidas y productos químicos $(15,9 \% / n=906)$. Se verificó tendencia creciente del coeficiente de mortalidad por suicidio del Estado para ambos sexos y para segmentos etarios de 20 a 39 años, 40 a 59 años y 60 o más años. Conclusión: El suicidio en Bahía predomina entre hombres jóvenes, con tendencia creciente del coeficiente total y en diferentes grupos poblacionales.

DESCRIPTORES: Suicidio; Mortalidad; Estudios de series temporales; Sistemas de información; Estudios ecológicos.

${ }^{1}$ Enfermeira. Mestre pelo Programa de Pós-Graduação em Enfermagem e Saúde da Universidade Estadual do Sudoeste da Bahia. Jequié, BA, Brasil.

${ }^{2}$ Enfermeira. Doutora em Enfermagem em Saúde Pública pela Escola de Enfermagem de Ribeirão Preto da Universidade de São Paulo. Professora Titular da Universidade Estadual do Sudoeste da Bahia. Jequié, BA, Brasil.

${ }^{3}$ Cirurgião dentista. Doutor em Odontologia Preventiva e Social pela Universidade Estadual Paulista Júlio de Mesquita Filho. Professor Titular da Universidade Estadual do Sudoeste da Bahia. Jequié, BA, Brasil. 


\section{INTRODUÇÃO}

O suicídio constitui uma das formas de violência autoinfligida, na qual o indivíduo intencionalmente causa a cessação da própria vida ${ }^{(1)}$. Configura-se em fenômeno complexo, com múltiplos fatores associados, que mudam de acordo com a cultura, o momento histórico, o grupo social, os aspectos biológicos, os psicológicos e ambientais, sendo considerado tema tabu em muitas sociedades ${ }^{(2)}$.

O comportamento suicida varia desde o pensamento em se matar até a elaboração de um plano e obtenção dos meios para a consumação do ato, e, atualmente, representa, no contexto mundial, importante problema de saúde pública ${ }^{(1)}$, tendo em vista o aumento no número de mortes nos últimos anos, tornando-se uma das dez principais causas de morte no mundo, a terceira mais frequente entre pessoas de 15 e 34 anos, além dos consequentes impactos sociais, econômicos, emocionais e pessoais $^{(1,3)}$.

Do ponto de vista econômico, o suicídio e suas tentativas representam enorme custo para a sociedade, em virtude dos investimentos necessários para a formação do indivíduo, dos anos potenciais de vida perdidos devido à morte precoce, e dos custos com atendimentos hospitalares ${ }^{(4)}$. No Brasil, em pesquisa publicada em 2007, o suicídio acarretou perda total de aproximadamente 1,3 bilhão, sendo registrado um valor médio de perda de capital humano de 163 mil por vítima, posicionando-se atrás apenas dos homicídios e dos acidentes de trânsito que apresentaram médias de 189,5 mil e 172 mil, respectivamente ${ }^{(5)}$.

No que se refere ao coeficiente de mortalidade por suicídio, no Brasil, este gira em torno de 6,2 mortes para 100.000 habitantes no ano ${ }^{(6)}$, valor considerado baixo quando comparado aos de outros países como França, China, Suíça, Bélgica, Áustria, Estados Unidos e os do Leste Europeu ${ }^{(7)}$. Entretanto, pelo fato de ser um país populoso, se ao invés do coeficiente de mortalidade for analisado o número absoluto de óbitos por essa causa, o Brasil ocupará o nono lugar numa escala mundial ${ }^{(8)}$.

No âmbito regional, no Sudeste brasileiro concentram-se $50 \%$ dos casos de suicídio, e no Sul encontram-se os maiores coeficiente de mortalidade por esse grupo de causa ${ }^{(9)}$. Todavia, ao avaliar a evolução da mortalidade por suicídio, as regiões Nordeste e Centro-Oeste apresentaram maior crescimento do coeficiente de mortalidade, exibindo um aumento de $130 \%$ e $68 \%$, respectivamente ${ }^{(3)}$.

Nesse contexto, evidencia-se que, embora o suicídio seja mais frequente nas áreas mais desenvolvidas do país, há um crescimento significativo nas regiões em desenvolvimento, a exemplo da região Nordeste, com destaque para os estados do Ceará e da Bahia, por representarem juntos mais de $40 \%$ das mortes por suicídio na região ${ }^{(10)}$.

Diante desse panorama, e considerando a escassez de estudos destinados à avaliação da mortalidade por suicídio no Estado da Bahia, de modo a possibilitar melhor compreensão da atual situação da problemática no Estado, assim como dos aspectos relacionados com a sua ocorrência nos diferentes grupos populacionais, o presente estudo tem como objetivo analisar a tendência temporal da mortalidade por suicídio no estado da Bahia, segundo sexo e faixa etária, no período de 1996 a 2013.

\section{- MÉTODOS}

Trata-se de um estudo epidemiológico, do tipo ecológico de série temporal, com dados secundários referentes à mortalidade por suicídio no estado da Bahia, no período de 1996 a 2013.

Os dados foram obtidos, junto com o Sistema de Informação sobre Mortalidade (SIM), por meio eletrônico, no portal do Departamento de Informação do Sistema Único de Saúde (DATASUS) do Ministério da Saúde. O período do estudo (1996 a 2013) foi determinado por corresponder aos anos de abrangência da atual Classificação Estatística Internacional de Doenças e Problemas Relacionados à Saúde, $10^{\mathrm{a}}$ revisão (CID-10), que enquadra o suicídio como "causas externas de morbimortalidade", sob os códigos X60 a X84. 
Para o estudo, considerou-se os óbitos que tinham como causa básica o suicídio, correspondentes ao grupo populacional com idade $\geq 10$ anos, residentes no estado da Bahia. Foram excluídos os casos cujos indivíduos possuíam idade menor que 10 anos, pois o estudo aponta que óbitos por suicídio nessa faixa etária são raros, e quando acontecem são de difícil caracterização, sendo na maioria das vezes classificados como causas acidentais ${ }^{(11)}$. Neste estudo foram identificados três óbitos por suicídio em menores de 10 anos, sendo estes excluídos da análise dos dados.

As variáveis analisadas no estudo foram: sexo (masculino; feminino); faixa etária (10 a 19 anos; 20 a 39 anos; 40 a 59 anos; e 60 anos ou mais); e as categorias da CID-10 correspondentes ao suicídio, agrupadas neste estudo como: autointoxicação intencional por medicamentos e substâncias não especificadas (X60-X64); autointoxicação intencional por álcool (X65); autointoxicação intencional por pesticidas e produtos químicos (X68-X69); lesão autoprovocada intencional por enforcamento e estrangulamento (X70); lesão autoprovocada intencional por afogamento/submersão (X71); lesão autoprovocada intencional por arma de fogo (X72-X74); lesão autoprovocada intencional por fumaça, fogo e gás (X75-X77); lesão autoprovocada intencional por arma branca e objetos contundentes (X78-X79); lesão autoprovocada intencional por precipitação de lugar elevado (X80); lesão autoprovocada intencional por meio não especificado (X84), e demais lesões autoprovocadas intencionalmente (X81-X83; X66-X67).

Foram calculados os coeficientes de mortalidade por suicídio total e os estratificados por sexo e faixa etária, sendo correspondentes a grupos de 100.000 habitantes. Os dados demográficos, base para os cálculos dos coeficientes, foram disponibilizados pela Fundação Instituto Brasileiro de Geografia e Estatística (IBGE), a partir do sítio do DATASUS. Os coeficientes foram padronizados por faixa etária, utilizando-se o método direto, adotando-se como padrão a população do estado da Bahia do ano de 2010. Julgou-se a padronização necessária para dispor de coeficientes de mortalidade que fossem comparáveis entre si ao longo do período estudado.

Para análise de tendência dos coeficientes (em logaritmos) foi utilizada a modelagem de regressão de Prais-Winsten, com intuito de corrigir os efeitos da autocorrelação serial ao longo do tempo. A partir da estimativa dos coeficientes beta 1 (b1). obtidos na regressão, calculou-se as variações percentuais anuais (APC: annual percent change) e os respectivos intervalos de confiança de $95 \%\left(\mathrm{IC}_{95 \%}\right)$, conforme método proposto por Antunes e Cardoso ${ }^{(12)}$. As tendências foram classificadas como crescentes $(p \leq$ 0,05 e coeficiente de regressão positivo), decrescentes ( $p \leq 0,05$ e coeficiente de regressão negativo) e estáveis $(p>0,05)$. Os valores de $p$ foram obtidos pelo Teste de Wald.

Para tabulação, análise descritiva, cálculo dos coeficientes e construção de figuras foi utilizado o programa Microsoft Office Excel 2010, e para análise de tendência o programa estatístico SPSS (Statiscal Package for the Social Sciences), versão 21.0.

Vale ressaltar que as bases de dados utilizadas neste estudo são de acesso público, nas quais existe a omissão da identificação dos indivíduos, o que atende aos preceitos éticos de pesquisa envolvendo seres humanos, segundo a Resolução n. 466 de 12 dezembro de 2012 do Conselho Nacional de Saúde ${ }^{(13)}$. Desse modo, dispensou a submissão ao Comitê de Ética em Pesquisa.

\section{RESULTADOS}

No período de 1996 a 2013 foram registrados 5.693 óbitos por suicídio envolvendo indivíduos com idade $\geq 10$ anos no estado da Bahia. 
Tabela 1 - Óbitos por suicídio por categorias da CID-10*, segundo sexo e faixa etária. Bahia, Brasil, 1996 a 2013

\begin{tabular}{|c|c|c|c|c|c|c|c|c|c|c|c|c|c|c|}
\hline \multirow{3}{*}{ CID-10* } & \multicolumn{6}{|c|}{ Sexo** } & \multicolumn{8}{|c|}{ Faixa Etária** } \\
\hline & \multicolumn{2}{|c|}{ Total } & \multicolumn{2}{|c|}{ Masculino } & \multicolumn{2}{|c|}{ Feminino } & \multicolumn{2}{|c|}{10 a 19} & \multicolumn{2}{|c|}{20 a 39} & \multicolumn{2}{|c|}{40 a 59} & \multicolumn{2}{|c|}{$\begin{array}{l}60 \text { ou } \\
\text { mais }\end{array}$} \\
\hline & $\mathbf{N}$ & $\%$ & $\mathbf{N}$ & $\%$ & $\mathbf{n}$ & $\%$ & $\mathbf{n}$ & $\%$ & $\mathbf{n}$ & $\%$ & $\mathbf{n}$ & $\%$ & $\mathbf{n}$ & $\%$ \\
\hline $\begin{array}{l}\text { Autointoxicação por } \\
\text { Medicamentos e sub. biológicas } \\
\text { NE }\end{array}$ & 144 & 2,5 & 83 & 1,8 & 61 & 5,8 & 19 & 4,7 & 72 & 2,7 & 45 & 2,5 & 8 & 0,9 \\
\hline Autointoxicação por álcool & 20 & 0,4 & 15 & 0,3 & 05 & 0,5 & - & - & 10 & 0,4 & 07 & 0,4 & 03 & 0,4 \\
\hline $\begin{array}{l}\text { Autointoxicação por pesticidas } \\
\text { e produtos químicos }\end{array}$ & 906 & 15,9 & 598 & 12,9 & 308 & 29,1 & 97 & 24 & 402 & 15,4 & 293 & 16,5 & 112 & 13,1 \\
\hline $\begin{array}{l}\text { Lesão autoprovocada por } \\
\text { enforcamento }\end{array}$ & 3336 & 58,6 & 2901 & 62,6 & 433 & 40,9 & 198 & 49 & 1484 & 56,7 & 1077 & 60,5 & 552 & 64,3 \\
\hline $\begin{array}{l}\text { Lesão autoprovocada por } \\
\text { afogamento/submersão }\end{array}$ & 83 & 1,5 & 54 & 1,2 & 29 & 2,7 & 9 & 2,3 & 37 & 1,4 & 23 & 1,3 & 12 & 1,6 \\
\hline $\begin{array}{l}\text { Lesão autoprovocada por arma } \\
\text { de fogo }\end{array}$ & 582 & 10,2 & 545 & 11,8 & 37 & 3,5 & 47 & 11,6 & 313 & 12 & 152 & 8,5 & 68 & 7,9 \\
\hline $\begin{array}{l}\text { Lesão autoprovocada por } \\
\text { dispositivo/explosivos }\end{array}$ & 82 & 1,4 & 36 & 0,8 & 46 & 4,3 & - & - & 30 & 1,1 & 34 & 1,9 & 26 & 2,1 \\
\hline $\begin{array}{l}\text { Lesão autoprovocada por arma } \\
\text { branca e objetos contundentes }\end{array}$ & 127 & 2,2 & 107 & 2,3 & 20 & 1,9 & 5 & 1,2 & 68 & 2,6 & 42 & 2,4 & 18 & 1,4 \\
\hline $\begin{array}{l}\text { Lesão autoprovocada por } \\
\text { precipitação de lugar elevado }\end{array}$ & 160 & 2,8 & 101 & 2,2 & 59 & 5,6 & 13 & 3,2 & 78 & 3 & 40 & 2,2 & 14 & 3 \\
\hline $\begin{array}{l}\text { Lesão autoprovocada por meio } \\
\text { NE }\end{array}$ & 200 & 3,5 & 153 & 3,3 & 47 & 4,4 & 14 & 3,5 & 98 & 3,7 & 55 & 3,1 & 33 & 3,9 \\
\hline Demais causas autoprovocadas & 53 & 1 & 39 & 0,8 & 14 & 1,3 & 02 & 0,5 & 27 & 1 & 12 & 0,7 & 12 & 1,4 \\
\hline TOTAL & 5693 & 100 & 4632 & 100 & 1059 & 100 & 404 & 100 & 2619 & 100 & 1780 & 100 & 858 & 100 \\
\hline
\end{tabular}

Fonte: Ministério da Saúde. Departamento de Informática do Sistema Único de Saúde. Sistema de Informação sobre Mortalidade.

* Classificação Estatística Internacional de Doenças e Problemas Relacionados à Saúde -10ª revisão

** Foram excluídos os casos ignorados.

NE: Não especificado.

Na Tabela 1 os óbitos foram distribuídos por categoria da CID-10, segundo sexo e faixa etária. Observou-se que 4.632 (81,4\%) eram do sexo masculino e 2.619 (46\%) do grupo etário de 20 a 39 anos. Quanto às categorias da CID-10, evidenciou-se tanto na análise total dos óbitos quanto na estratificada por sexo e faixa etária maior proporção de casos que tiveram as lesões autoprovocadas intencionalmente por enforcamento e estrangulamento como causa da morte: 3.336 (58,6\%) casos no total; 2.901 (62,6\%) do sexo masculino; 433 (40,9\%) do sexo feminino; 198 (49\%) de 10 a 19 anos; 1.484 (56,7\%) de 20 a 39 anos: 1.077 (60,5\%) de 40 a 59 anos; e $552(64,3 \%)$ de 60 anos ou mais; seguidas pela autointoxicação por pesticidas e produtos químicos: 906 (15,9\%) casos no total; 598 (12,9\%) do sexo masculino, 308 (29,1\%) do sexo feminino; 97 (24\%) de 10 a 19 anos; 402 (15,4\%) de 20 a 39 anos; 293 (16,5\%) de 40 a 59 anos; e $112(13,1 \%)$ de 60 anos ou mais.

No que se refere à evolução dos coeficientes de mortalidade por suicídio no Estado, conforme demonstram a Figura 1 e a Tabela 2, notou-se crescimento do coeficiente de mortalidade por esta causa (102,9\%), que passou de 2,03 por 100.000 habitantes em 1996 para 4,12 por 100.000 habitantes em 2013, apresentando incremento anual de 12,1\% e tendência crescente para o período analisado $(\mathrm{p}<0,001)$ (Tabela 3). 


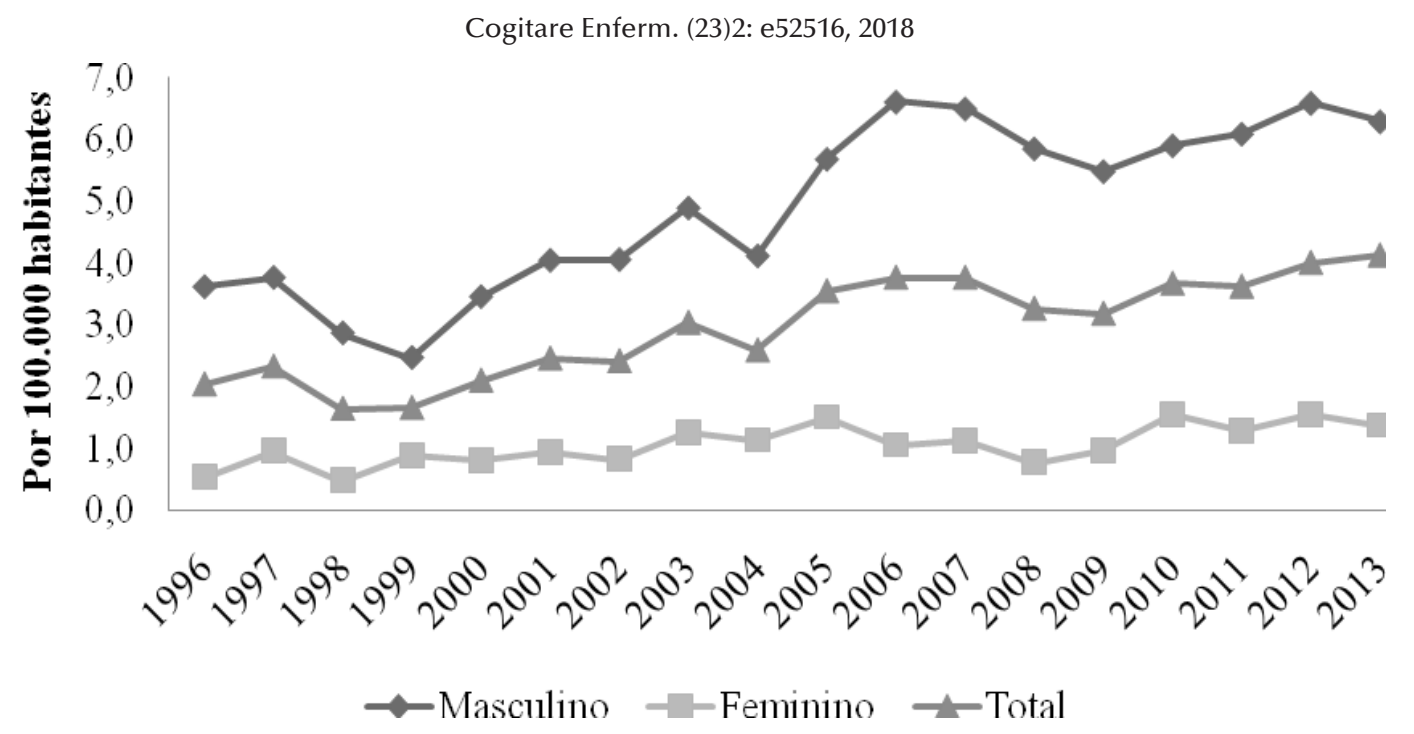

Figura 1- Evolução dos coeficientes* de mortalidade por suicídio total e estratificado por sexo. Bahia, Brasil, 1996 a 2013

Fonte: MS/SVS/DATASUS/SIM; IBGE.

*Coeficiente por 100.000 habitantes padronizados pelo método direto por faixa etária.

Tabela 2 - Evolução do coeficiente* de mortalidade por suicídio total e estratificados, segundo sexo e faixa etária. Bahia, Brasil, 1996 a 2013

\begin{tabular}{|c|c|c|c|c|c|c|c|c|c|c|c|c|c|c|c|c|c|c|}
\hline \multirow[b]{2}{*}{ Variáveis } & \multicolumn{18}{|c|}{ Ano } \\
\hline & 1996 & 1997 & 1998 & 1999 & 2000 & 2001 & 2002 & 2003 & 2004 & 2005 & 2006 & 2007 & 2008 & 2009 & 2010 & 2011 & 2012 & 2013 \\
\hline Total & 2,03 & 2,32 & 1,64 & 1,65 & 2,10 & 2,45 & 2,41 & 3,03 & 2,58 & 3,54 & 3,76 & 3,76 & 3,26 & 3,18 & 3,66 & 3,62 & 4 & 4,12 \\
\hline \multicolumn{19}{|l|}{ Sexo } \\
\hline Masculino & 3,61 & 3,76 & 2,86 & 2,46 & 3,45 & 4,04 & 4,05 & 4,89 & 4,11 & 5,68 & 6,61 & 6,50 & 5,85 & 5,48 & 5,90 & 6,09 & 6,59 & 6,29 \\
\hline Feminino & 0,53 & 0,95 & 0,47 & 0,88 & 0,80 & 0,93 & 0,82 & 1,25 & 1,12 & 1,50 & 1,04 & 1,11 & 0,77 & 0,96 & 1,54 & 1,27 & 1,54 & 1,36 \\
\hline \multicolumn{19}{|l|}{ Faixa etária } \\
\hline 10 a 19 & 0,54 & 0,44 & 0,53 & 0,46 & 0,65 & 0,74 & 0,89 & 0,69 & 0,75 & 0,77 & 0,58 & 1,01 & 0,63 & 1,02 & 1,20 & 0,93 & 1,11 & 0,07 \\
\hline 20 a 39 & 2,10 & 2,67 & 1,68 & 1,79 & 1,86 & 2,47 & 2,76 & 3,18 & 2,70 & 3,89 & 4,58 & 4,46 & 3,31 & 3,27 & 3,50 & 4,47 & 4,89 & 3,73 \\
\hline 40 a 59 & 3,18 & 2,68 & 2,24 & 2,22 & 3,22 & 3,88 & 3,12 & 4,68 & 3,83 & 4,73 & 5,17 & 4,46 & 4,77 & 4,40 & 4,82 & 4,38 & 4,94 & 5,22 \\
\hline 60 ou mais & 2,23 & 3,89 & 2,40 & 2,27 & 3,34 & 2,76 & 2,65 & 3,81 & 3,06 & 5,15 & 4,05 & 5,04 & 4,78 & 4,36 & 4,34 & 3,91 & 4,51 & 6,83 \\
\hline
\end{tabular}

Fonte: MS/SVS/DATASUS/SIM; IBGE.

*Coeficiente por 100.000 habitantes padronizados pelo método direto por faixa etária.

Do mesmo modo, constatou-se tendência crescente para o coeficiente de mortalidade por suicídio no sexo masculino, que apresentou aumento percentual de $11,8 \%$ ao ano $\left(\mathrm{IC}_{95 \%}: 5,9 ; 18\right)$, passando de 3,61 por 100.000 habitantes em 1996 para 6,29 por 100.000 habitantes em 2013. No sexo feminino, observouse aumento percentual anual de 9,8\%, com coeficiente que passou de 0,53 por 100.000 habitantes em 1996 para 1,36 por 100.000 habitantes em 2013, e tendência crescente no período investigado $(p=0,002)$ (Figura 1 e Tabelas 2 e 3). 


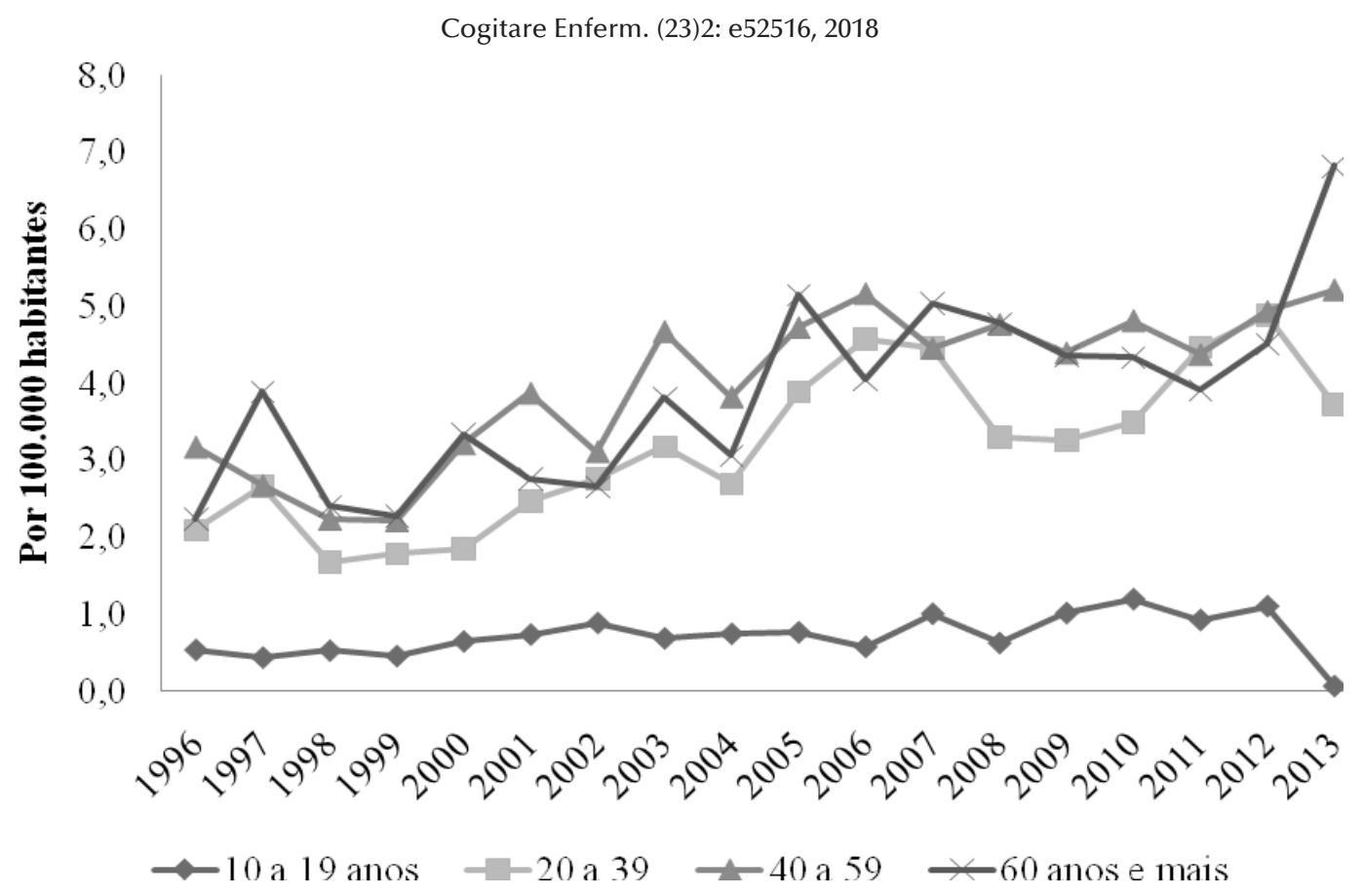

Figura 2 - Evolução dos coeficientes* de mortalidade por suicídio, segundo faixa etária. Bahia, Brasil, 1996 a 2013 Fonte: MS/SVS/DATASUS/SIM; IBGE.

*Coeficiente por 100.000 habitantes padronizados pelo método direto por faixa etária.

Na Figura 2 e na Tabela 2 está descrita a evolução dos coeficientes de mortalidade por suicídio, segundo a faixa etária. Evidenciou-se tendência crescente para os grupos etários de 20 a 39 anos (2,10/100.00 em 1996 e 3,73/100.000 em 2013), 40 a 59 anos (3,18/100.000 em 1996 e 5,22/100.000 em 2013) e naqueles com 60 anos ou mais (2,23/100.000 em 1996 e 6,83/100.000 em 2013), com variação percentual anual de $11,8 \%\left(\mathrm{IC}_{95 \%}: 5,4 ; 18,7\right), 9,9 \%\left(\mathrm{IC}_{95 \%}: 5 ; 15\right)$ e $11 \%\left(\mathrm{IC}_{95 \%}: 6,9 ; 15,2\right)$, respectivamente. Quanto ao grupo etário de 10 a 19 anos, este apresentou os menores coeficientes de mortalidade por suicídio, com comportamento estável ao longo do período $(p=0,729)$ (Tabela 3$)$.

Tabela 3 - Resultado da análise de tendência do coeficiente de mortalidade por suicídio no estado da Bahia, Brasil, 1996 a 2013

\begin{tabular}{lcccc}
\hline & APC $_{\%}$ & IC $_{95 \%}$ & p-valor* & Tendência \\
\hline Total & 12,1 & 7,$3 ; 17,1$ & $<0,001$ & Crescente \\
\hline Sexo & & & & \\
\hline Masculino & 11,8 & 5,$9 ; 18$ & 0,001 & Crescente \\
\hline Feminino & 9,8 & 4,$2 ; 15,6$ & 0,002 & Crescente \\
\hline Faixa etária & 2 & & & Estável \\
\hline 10 a 19 & 11,8 & $-9,5 ; 14,9$ & 0,729 & Crescente \\
\hline 20 a 39 & 9,9 & 5,$4 ; 18,7$ & 0,001 & Crescente \\
\hline 40 a 59 & 11 & 5,$0 ; 15$ & $<0,001$ & Crescente \\
\hline 60 ou mais & 6,$9 ; 15,2$ & $<0,001$ & & \\
\hline
\end{tabular}

APC: anual percent change ou variação percentual anual.

$\mathrm{IC}_{95 \%:}$ Intervalo de Confiança de $95 \%$.

*Teste de Wald obtido por meio de regressão de Prais-Winsten. 


\section{DISCUSSÃO}

Os resultados deste estudo apontam a tendência crescente do coeficiente de mortalidade por suicídio na Bahia, com aumento de 102,9\% no período analisado e variação anual de 12,1\%. Este achado demonstra que o Estado apresenta maior crescimento do coeficiente quando comparado ao estimado para o Brasil, no período de 2000 a $2012(26,5 \%)^{(6)}$ e para o Estado do Espírito Santo, nos anos de 1980 a $2006(24,4 \%)^{(14)}$.

Sobre este aspecto, ressalta-se que o crescimento evidenciado pode ser reflexo não apenas do aumento do número de casos, mas também de possíveis melhorias no preenchimento das declarações de óbitos, em virtude da melhor identificação e classificação do suicídio como causa do óbito.

Ao avaliar a mortalidade por suicídio de acordo com o sexo, constatou-se que na Bahia, o ato suicida predomina no sexo masculino $(81,4 \%)$, com tendência crescente para o coeficiente de mortalidade. Estes resultados estão em conformidade com os apontados para o Brasil( ${ }^{(6)}$, outros Estados ${ }^{(14-16)}$ e capitais brasileiras $^{(17-18)}$, assim como para outros países da América do Sul, como Argentina ${ }^{(19)}$ e Chile ${ }^{(19)}$.

A disparidade na ocorrência do suicídio entre os sexos é atribuída, geralmente, a uma maior agressividade, competitividade, impulsividade e ao maior acesso as tecnologias letais pelos homens, o que aumenta a probabilidade de terem sucesso no ato ${ }^{(14-15)}$. Ademais, o alcoolismo, acompanhado dos seus danos, é referido como outro fator que leva o indivíduo a tirar a própria vida, o que também pode justificar a maior proporção de casos do sexo masculino, como exemplo em pesquisa em Santa Catarina, com média de $80 \%$ de alcoolistas homens ${ }^{(16)}$.

Apesar da menor frequência entre os óbitos, o sexo feminino apresentou tendência crescente para o coeficiente de mortalidade por suicídio, o que diverge dos resultados apontados para outros Estados brasileiros como Espírito Santo ${ }^{(14)}$ e São Paulo(15), onde os coeficientes mantiveram-se estáveis, e do Rio Grande do Sul ${ }^{(20)}$, onde foi verificada tendência decrescente. Uma possível explicação para a tendência encontrada na Bahia seria o reflexo de um sistema fragilizado na identificação precoce da pessoa em risco suicida, uma vez que é demonstrado por vários estudos que embora o suicídio seja mais frequente entre os homens, as tentativas predominam entre as mulheres ${ }^{(4,16)}$.

No que se refere à distribuição dos óbitos por faixa etária, notou-se maior proporção de adultos jovens (20 a 39 anos), seguidos dos adultos em idade mais avançada (40 a 59 anos), com tendência crescente no coeficiente de mortalidade para ambos os grupos. Estes resultados corroboram os descritos em estudos realizados nos estados do Espírito Santo ${ }^{(14)}$ e São Paulo ${ }^{(15)}$, e em municípios como Belo Horizonte/MG ${ }^{(18)}$, Itabira/MG ${ }^{(18)}$ e Independência/CE ${ }^{(21)}$.

Outrossim, os indivíduos com 60 anos ou mais também apresentaram destaque na mortalidade por suicídio no Estado, com aumento de $11 \%$ ao ano no coeficiente e tendência crescente no período analisado. Estas características evolutivas diferem das encontradas para o Brasil entre os anos de 2000 e 2012, onde o crescimento da mortalidade por suicídio foi maior entre os adultos de 25 e 59 anos e nos jovens de 10 a 24 anos $^{(6)}$, assim como para o estado de São Paulo no período de 1996 a 2009 ${ }^{(15)}$, onde a mortalidade por suicídio em idosos apresentou declínio. Não obstante, estudo demonstrou que semelhante à Bahia outros estados das regiões Norte e Nordeste apresentaram tendência crescente para a mortalidade por suicídio em pessoas com 60 anos ou mais ${ }^{(22)}$.

Estas diferenças e mudanças no perfil de mortalidade por suicídio ao longo do tempo podem estar relacionadas com as modificações no contexto social, no qual o indivíduo encontra-se inserido, uma vez que os motivos que induzem o ato suicida diferem entre os grupos de idade ${ }^{(6)}$. Entre jovens, por exemplo, as razões geralmente são afetivas, como rompimentos de relacionamentos, pressões acadêmicas e ocupacionais devido à competitividade no mercado de trabalho ${ }^{(6)}$. Nos adultos e pessoas de meia idade, os aspectos relacionados com o trabalho e as cobranças do âmbito familiar são os fatores de risco mais expressivos, sobretudo no sexo masculino ${ }^{(6)}$.

Já entre os idosos, os episódios suicidas podem estar relacionados com as dificuldades em lidar com o processo de envelhecimento e suas consequências, como isolamento social, morte de uma pessoa querida (comumente o cônjuge), doença terminal com dores incontroláveis, medo do prolongamento da vida sem dignidade, além das modificações nos papeis sociais que lhes conferiam reconhecimento e as situações de dependência física/mental diante das quais o idoso se sente humilhado ${ }^{(23)}$. 
Sobre os meios empregados no ato suicida, destacaram-se neste estudo o enforcamento e estrangulamento, seguidos da autointoxicação por pesticidas e produtos químicos, tanto na análise total dos óbitos quanto na estratificada por sexo e faixa etária. Estes métodos são semelhantes aos referidos como principais causas de suicídio no Brasil ${ }^{(3,6)}$ e em outros estados do país ${ }^{(16,20)}$.

A facilidade no acesso ao método escolhido tem sido apontada em diversos estudos como importante fator de risco para a ocorrência do evento. Assim, estratégias que dificultem a acessibilidade, a exemplo das medidas de proteção em frascos de medicamentos, maior controle e fiscalização no porte de armas de fogo e na comercialização ilegal de pesticidas e de outros produtos químicos podem auxiliar na redução dos $\operatorname{casos}^{(24)}$.

Alguns métodos, como o enforcamento e estrangulamento são de difícil controle. Nestes casos, a identificação precoce da pessoa em risco, por parte dos profissionais da saúde e serviços especializados, torna-se crucial para a promoção da assistência em saúde mental, assim como para orientação de familiares e pessoas próximas à vítima acerca da vigilância e identificação dos momentos emergenciais, com vista a impedir a tentativa do suicídio ${ }^{(6)}$.

No que se refere ao controle e à prevenção, no Brasil o suicídio já é reconhecido como um problema de saúde pública, tendo sido adotadas algumas medidas pelo Ministério da Saúde, como a criação da Estratégia Nacional de Prevenção ao Suicídio, através da Portaria n. ${ }^{\circ} 2.542 / 2005^{(25)}$, instituição das Diretrizes Nacionais para Prevenção do Suicídio, a serem implantadas em todas as unidades federativas, por meio da Portaria n. ${ }^{\circ} 1.876 / 2006^{(26)}$ e a publicação do Manual de Prevenção do Suicídio para Profissionais das Equipes de Saúde Mental(27).

Associadas às políticas voltadas para prevenção do suicídio, também são vigentes no Brasil as políticas públicas de saúde mental, que estabelecem os Centros de Atenção Psicossocial (CAPS) como dispositivos de referência na área. Contudo, esses serviços ainda não executam ações direcionadas à prevenção do suicídio(6).

Apesar do avanço nas legislações referentes à temática, e reconhecimento por parte das entidades federativas acerca da magnitude do problema, ainda não se efetivou na prática um programa específico que promova ações preventivas para o suicídio. Em vista disso, o ideal seria o investimento na implantação de serviços especializados e no treinamento de profissionais que auxiliassem no rastreamento de pessoas em sofrimento mental e em situação de risco suicida.

Como limitações deste estudo, pode-se citar a utilização de dados extraídos do SIM, que possui como principal entrave a possível ocorrência do subregistro e/ou falhas no preenchimento das declarações de óbito. Ademais, a possibilidade de subnotificação de casos, que pode estar relacionada com o estigma social, as razões jurídicas, a recusa do ato suicida por questões religiosas e, ainda, a relutância ou o preenchimento inadequado pelo profissional médico, ao registrar o óbito como "morte acidental" ou "causa indeterminada"(28) pode ter subestimado o quantitativo de casos.

Embora existam tais limitações, o SIM representa uma potencial ferramenta para os estudos de tendência temporal, pois dispõe de dados oficiais, que dentre outras atribuições, podem direcionar as ações e a elaboração de políticas públicas.

\section{- CONCLUSÃo}

No presente estudo, verificou-se tendência crescente para o coeficiente de mortalidade por suicídio na Bahia, em ambos os sexos, bem como nos grupos etários de 20 a 39 anos, 40 a 59 anos e naqueles com 60 anos ou mais.

Estes resultados apontam a imprescindibilidade da capacitação de profissionais da saúde na identificação de pessoas com risco suicida, e que em parceria com a rede de atendimento à saúde mental possam promover o desenvolvimento de ações preventivas fundamentadas na lógica de que cada indivíduo tem suas particularidades e sofre influências do contexto social onde se encontra inserido. 


\section{- REFERÊNCIAS}

1. Krug EG, Dahlberg LL, Mercy JA, Zwi AB, Lozano R. World Report on Violence and Health. [Internet] Geneva: WHO; 2002 [acesso em 18 jan 2017]. Disponível: http://apps.who.int/iris/bitstream/10665/42495/1/9241545615_ eng.pdf.

2. Ministério da Saúde (BR). Organização Pan-Americana da Saúde (OPAS/OMS). Secretária de Gestão Estratégica Parcipativa. Departamnto de Monitoramento e Avaliação da Gestão do SUS. Temático Prevenção de Violência e Cultura da Paz III - Painel de indicadores do SUS nº 5. [Internet] Brasília: Ministério da Saúde/OPAS; 2008 [acesso em 20 de fev de 2017]. Disponível: http://bvsms.saude.gov.br/bvs/publicacoes/painel_indicadores_sus_ n5_p1.pdf.

3. Lovisi GM, Santos AS, Legay L, Abelha L, Valencia E. Epidemiological analysis of suicide in Brazil from 1980 to 2006. Rev. Bras. Psiquiatr. [Internet] 2009;31(Suppl 2) [acesso em 23 abr 2017]. Disponível: http://dx.doi. org/10.1590/S1516-44462009000600007.

4. Souza VS, Alves MS, Silva LA, Lino DCSF, Nery AA, Casotti CA. Tentativas de suicídio e mortalidade por suicídio em um município no interior da Bahia. Rev. Bras. Psiquiatr. [Internet] 2011;60(4) [acesso em 07 jan 2017 ]. Disponível: http://dx.doi.org/10.1590/S0047-20852011000400010.

5. Cerqueira DRC, Carvalho AXY, Lobão WJA, Rodrigues RI. Ministério do Planejamento, Orçamento e Gestão. Instituto de Pesquisa Econômica Aplicada (IPEA). Análise dos custos e consequências da violência no Brasil. Texto para Discussão no 1284. [Internet] Brasília: IPEA; 2007 [acesso em 3 mar 2017]. Disponível: http://www.ipea. gov.br/portal/images/stories/PDFs/TDs/td_1284.pdf.

6. Machado DB, dos Santos DN. Suicídio no Brasil, de 2000 a 2012. J. Bras. Psiquiatr. [Internet] 2015;64(1) [acesso em 07 jan 2017]. Disponível: http://dx.doi.org/10.1590/0047-2085000000056.

7. Varnik P, Sisask M, Varnik A, Arensman E, Van Audenhove C, Van der Feltz-Cornelis C, et al. Validity of suicide statistics in Europe in relation to undetermined deaths: Developing the 2-20 benchmark. Inj prev. [Internet] 2012;18(5) [acesso em 07 jan 2017]. Disponível: http://dx.doi.org/10.1136/injuryprev-2011-040070.

8. Associação Brasileira de Psiquiatria (ABP). Comportamento suicida: conhecer para prevenir. Dirigido para profissionais de imprensa. [Internet] São Paulo: ABP Editora; 2009 [acesso em 21 jun 2017]. Disponível: http:// www.cvv.org.br/downloads/cartilha_suicidio_profissionais_imprensa.pdf.

9. Organização Mundial de Saúde(OMS). Departamento de saúde mental. Transtornos mentais e comportamentais. Prevenção do suicídio: um manual para médicos clínicos gerais. [Internet] Genebra: OMS; 2000 [acesso em 14 mar 2017]. Disponível: http://apps.who.int/iris/bitstream/10665/67165/7/WHO_MNH_MBD_00.1_por.pdf.

10. Ministério da Saúde (BR). Departamento de Informação do SUS (DATASUS). Informações de saúde. Óbitos por causas externas - Brasil. [Internet] 2002 a 2012 [acesso em 14 nov 2016]. Disponível: http://tabnet.datasus.gov. br/cgi/tabcgi.exe?sim/cnv/ext10uf.def.

11. Monteiro RA, Bahia CA, Paiva EA, de Sá NNB, Minayo MCS. Hospitalizações relacionadas a lesões autoprovocadas intencionalmente - Brasil, 2002 a 2013. Ciênc. saúde coletiva. [Internet] 2015;20(3) [acesso em 20 jan 2017]. Disponível: http://dx.doi.org/10.1590/1413-81232015203.16282014.

12. Antunes JLF, Cardoso MRA. Uso da análise de séries temporais em estudos epidemiológicos. Epidemiol. Serv. Saúde. [Internet] 2015;24(3) [acesso em 20 jun 2017]. Disponível: http://dx.doi.org/10.5123/S167949742015000300024.

13. Ministério da Saúde (BR). Conselho Nacional de Saúde. Diretrizes e normas regulamentadoras de pesquisa envolvendo seres humanos. Resolução n. 466, de 12 de dezembro de 2012. Brasília; 2012.

14. Macente LB, Zandonade E. Estudo da série histórica de mortalidade por suicídio no Espírito Santo (de 1980 a 2006). J. Bras. Psiquiatr. [Internet] 2011;60(3) [acesso em 19 jun 2017]. Disponível: http://dx.doi.org/10.1590/S004720852011000300001. 
15. Bando DH, Brunoni AR, Fernandes TG, Benseñor IM, Lotufo PA. Suicide rates and trends in São Paulo, Brazil, according to gender, age and demographic aspects: a joinpoint regression analysis. Rev. Bras. Psiquiatr. [Internet] 2012;34(3) [acesso em 21 jun2017]. Disponível: http://dx.doi.org/10.1016/j.rbp.2012.02.001.

16. Sehnem SB, Palosqui V. Características epidemiológicas do suicídio no estado de Santa Catarina. Fractal, Rev. Psicol. [Internet] 2014;26(2) [acesso em 20 mar 2017]. Disponível: http://dx.doi.org/10.1590/1984-0292/624.

17. Schnitmana G, Kitaoka EG, Arouca GSS, Lira ALS, Nogueira D, Duarte MB. Taxa de mortalidade por suicídio e indicadores socioeconômicos nas capitais brasileiras. Rev. Baiana Saúde Pública. [Internet] 2010;34(1) [acesso em 13 fev 2017]. Disponível: http://rbsp.sesab.ba.gov.br/index.php/rbsp/article/view/12.

18. de Souza ER, Minayo MCS, Cavalcante FG. O impacto do suicídio sobre a morbimortalidade da população de Itabira. Ciênc. saúde coletiva. [Internet] 2007;11(Suppl 0) [acesso em 20 jun 2017]. Disponível: http://dx.doi. org/10.1590/S1413-81232006000500022.

19. Quinlan-Davidson M, Sanhueza A, Espinosa I, Escamilla-Cejudo JA, Maddaleno M. Suicide Among Young People in the Americas. Journal of Adolescent Health. [Internet] 2014;54(3) [acesso em 21 jun 2017]. Disponível: https://doi.org/10.1016/j.jadohealth.2013.07.012.

20. Meneghel SN, Victora CG, Faria NMX, de Carvalho LA, Falk JW. Epidemiological aspects of suicide in Rio Grande do Sul, Brazil. Rev. Saúde Pública. [Internet] 2004;38(6) [acesso em 21 jun de 2017]. Disponível: http:// dx.doi.org/10.1590/S0034-89102004000600008.

21. Pordeus AMJ, Cavalcanti LPG, Vieira LJES, Coriolano LS, Osório MM, de Ponte MSR, et al. Tentativas e óbitos por suicídio no município de Independência, Ceará, Brasil. Ciênc saúde coletiva. [Internet] 2009;14(5) [acesso em 22 jun 2017]. Disponível: http://dx.doi.org/10.1590/S1413-81232009000500014.

22. Pinto LW, Pires TO, da Silva CMFP, de Assis SG. Suicide mortality trends in people aged 60 years or more in the Brazilian states: 1980 to 2009. Ciênc. saúde coletiva. [Internet] 2012;17(8) [acesso em 17 abr 2017]. Disponível: http://dx.doi.org/10.1590/S1413-81232012000800008.

23. Minayo MCS, Cavalcante FG. Suicide in elderly people: a literature review. Rev. Saúde Pública. [Internet] 2010;44(4) [acesso em 09 mar 2017]. Disponível: http://dx.doi.org/10.1590/S0034-89102010000400020.

24. Santos AS, Legay LF, Lovisi GM. Substâncias tóxicas e tentativas e suicídios: considerações sobre acesso e medidas restritivas. Cad. saúde colet. [Internet] 2013;21(1) [acesso em 02 abr 2017]. Disponível: http://dx.doi. org/10.1590/S1414-462X2013000100009.

25. Ministério da Saúde (BR). Portaria n. 2.542/GM de 22 de dezembro de 2005. Institui Grupo de Trabalho com o objetivo de elaborar e implantar a Estratégia Nacional de Prevenção ao Suicídio. Diário Oficial da União, [Internet] 22 dez 2005 [acesso em 21 jun 2017]. Disponível: http://bvsms.saude.gov.br/bvs/saudelegis/gm/2005/ prt2542_22_12_2005.html.

26. Ministério da Saúde (BR). Portaria n. 1.876 de 14 de agosto de 2006. Dispõe sobre as Diretrizes Nacionais para a Prevenção do Suicídio. Diário Oficial da União, [Internet] 14 ago 2006 [acesso em 21 jun 2017]. Disponível: http://bvsms.saude.gov.br/bvs/saudelegis/gm/2006/prt1876_14_08_2006.html.

27. Ministério da Saúde (BR). Organização Pan-Americana da Saúde (OPAS/OMS). Universidade Estadual de Campinas (UNICAMP). Prevenção do Suicídio: manual dirigido a profissionais das equipes de saúde mental. [Internet] Brasília: Ministério da Saúde/OPAS/OMS/UNICAMP; 2006 [acesso em 21 jun 2017]. Disponível: http:// www.cvv.org.br/downloads/manual_prevencao_suicidio_profissionais_saude.pdf.

28. Volpe FM, Corrêa H, Barrero SP. Epidemiologia do suicídio. In: Correa H, Barrero SP, organizadores. Suicídio: uma morte evitável. São Paulo: Atheneu; 2006. p.10-27. 